\title{
Design for Generating Chaos from a Class of Switching Linear Systems
}

\author{
Zhang Yuping*, Zhu Hong and Zeng Yong \\ School of Automation Engineering, University of Electronic Science and Technology of China, 610054 Chengdu, China
}

Received: 16 Mar. 2013, Revised: 17 Jul. 2013, Accepted: 19 Jul. 2013

Published online: 1 Mar. 2014

\begin{abstract}
In this paper, a systematic design approach based on event driven method is investigated for a class of switching systems, and the switching control system is able to generate chaos at will from linear systems under the designed switching rules. To gain this target, structures of a class of linear switching systems are analyzed properly, as a consequence of analysis, the corresponding criteria is obtained to judge the existence for this class of switching systems to generate chaos. Then, based on the analysis of structure, feedback controllers designing procedure of some non-autonomous switching systems is deduced step by step. Two numerical examples are given to illustrate the generated chaotic dynamic behavior of the systems, and the validity of main results is also verified by the simulations. Finally, a circuit is provided to show the potential applications of the approach in engineering.
\end{abstract}

Keywords: Chaotification, Switching Rules, State Feedback

\section{Introduction}

During the last decade, chaos has been extensively studied not only in scientific field, but also in engineering. Generally speaking, there are two main streams on chaos researching. On the one hand, people explored methods to avoid chaotic conditions when it is harmful. On the other hand, many researchers and engineers endeavored to find the ways to generate chaos. It comes from the fact, more and more studies show that chaos is helpful and beneficial, especially in some engineering fields such as liquid mixing, industrial electronics, secure communications, resonance prevention in mechanical systems, so chaos constructing attracts much attention in these years. Some new chaotic systems have been developed $[1,2,3,4]$.

In this paper, we present a new approach to construct chaos by using switching rule designing methods for a class of 2-dimension linear switching systems. As is well known, switching systems, one of the most important branch of hybrid system, are characterized by " $1+1 \neq 2$ " [5], and variety of dynamic behavior emerges by different switching rules. But researchers prefer to pay more attention to focus on stability analysis $[6,7,8]$. In this paper, we do some work to illustrate the diversity of its dynamic activities in another perspective. A new chaotification method is proposed, it will be analyzed by theoretical inference and circuit experiment. Two numerical examples and corresponding portraits are given to show the validity of the approach we got.

The paper is structured as follows. In the Section 2, switching rules based on event-dirven method will be designed, and corresponding feedback controllers will be provided together to generate chaos. In Section 3, 2 numerical examples are given to illustrate the chaotification, and the realization of a chaotic system by means of circuit is built in Section 4. Finally, a conclusion is given in Section 5.

\section{The Switching Control Chaotification}

We consider a class of linear switching control systems described by equations of the form:

$$
\dot{x}(t)=A_{\sigma} x(t)+u(x), x\left(t_{0}\right)=x_{0}
$$

where $x(t) \in \mathbb{R}^{2}$ is the state vector, $t_{0} \geq 0$ is the initial time and $x_{0}$ is the initial state. $\sigma \in \bar{m}=\{1,2, \ldots, N\}$, and $N>1$, is a piecewise constant function of time, named switching signal. It is dependent on continuous system state, i.e. the switching activity from one subsystem to another subsystem might happen once the state $x(t)$

\footnotetext{
*Corresponding author e-mail: zyp_002@163.com
} 
reaches predefined boundaries. $A_{i} \in \mathbb{R}^{2 \times 2}$ are a set of constant matrices describing the subsystems. And $u(x) \in \mathbb{R}^{2}$ is control input vector.

\subsection{Dynamical Analysis of the Autonomous systems}

In this section, we focus on a class of linear switching systems with specific structure. And a switching rule is designed to generate chaos or chaos-like behaviors.

Firstly, considering the corresponding autonomous system

$$
\dot{x}(t)=A_{\sigma} x(t), x\left(t_{0}\right)=x_{0}
$$

Let

$$
A_{i}=\left[\begin{array}{ll}
a_{i} & b_{i} \\
c_{i} & d_{i}
\end{array}\right]
$$

and $A_{i} \neq k A_{j}, k \in R /\{0\}, b_{i} c_{i}<0, b_{i} b_{j}>0, \Delta=\left(a_{i}-\right.$ $\left.d_{i}\right)^{2}+4 b_{i} c_{i}<0, i, j \in \bar{m}$.

Remark 1. Under the structure above, we can check that states orbits of subsystems spin in the same direction.

It is easy to obtain that each of the subsystems in (2) is non chaotic due to the property of linear system. To make system (2) chaotic, we have the following result. Proposition 2.1. If there exists a Hurwitz linear convex combination of $A_{i}$, i.e.

$$
\bar{A}=\sum_{i=1}^{m} \alpha_{i} A_{i}, \alpha_{1}, \cdots, \alpha_{m} \in(0,1), \sum_{i=1}^{m} \alpha_{i}=1
$$

and a symmetric matrix $P>0$, such that the following linear matrix inequality(LMI) holds

$$
\bar{A}^{T} P+P \bar{A}<0
$$

Then there exists at least one switching rule that can make the switching system generating chaos or chaos-like behavior.

Proof. We will illustrate the result above by steps followed.

Step 1.We separate the region of the plane as:

$$
\Omega_{i}=\left\{x \mid x^{T}(t)\left(A_{i}^{T} P+P A_{i}\right) x(t)<0\right\}
$$

We will clarify that

$$
\bigcup_{i=1}^{m} \Omega_{i}=R^{2} /\{0\}
$$

respect to the separation above.

If it doesn't hold. there must exist a region $D \in R^{2}$ satisfying

$$
D=R^{2} /\left(\left(\bigcup_{i=1}^{m} \Omega_{i}\right) \bigcup\{0\}\right)
$$

for any $x(t) \in D$, the inequalities

$$
x^{T}(t)\left(A_{i}^{T} P+P A_{i}\right) x(t) \geq 0
$$

holds for all $i \in \bar{m}$. But $\alpha_{i} \in(0,1), \sum_{i=1}^{m} \alpha_{i}=1$, that

$$
\sum_{i=1}^{m} \alpha_{i} x^{T}(t)\left(A_{i}^{T} P+P A_{i}\right) x(t) \geq 0
$$

Then

$$
x^{T}(t)\left(\bar{A}^{T} P+P \bar{A}\right) x(t) \geq 0
$$

It conflicts with (3), so (5) holds.

To avoid overlap, we reconstruct the separation as follow.

$$
\hat{\Omega}_{i j}=\Omega_{i} \bigcap \Omega_{j}, i<j, i, j \in \bar{m}
$$

$$
\hat{\Omega}_{i}=\Omega_{i}-\sum_{i \neq j, i, j \in \bar{m}} \hat{\Omega}_{i j}
$$

Then

$$
\begin{gathered}
\left(\bigcup_{i=1}^{m} \hat{\Omega}_{i}\right) \bigcup\left(\bigcup_{i<j, i, j \in \bar{m}} \hat{\Omega}_{i j}\right)=R^{2} /\{0\} \\
\hat{\Omega}_{i} \bigcap \hat{\Omega}_{j}=\varnothing, i \neq j, i, j \in \bar{m}
\end{gathered}
$$

Step 2.After separating the plan into several regions, we can design such switching rule as below:

Case 1: If $x(t) \in \hat{\Omega}_{i}$, and $g(x)<L(t)$, then the jth subsystem is activated $(j \neq i)$;

Case 2: If $x(t) \in \hat{\Omega}_{i} \cup \hat{\Omega}_{i j}$, and $g(x) \geq L(t)$, then the ith subsystem is activated;

Case 3: If $x(t) \in \hat{\Omega}_{i j}$, and $g(x)<L(t)$, then, if there exists other subsystems like $l(l \neq j \neq i)$, the lth subsystem is activated; otherwise, the ith subsystem is activated.

Among them, $g(x)>0$ is a distance function, $L(t)$ is a class of trigger functions, sawtooth function and sine function are possible choices. And $L(t) \leq M, M$ is a finite constant.

Remak 2. The choice of jth subsystem in case 1 can be designed as $j=\min \left\{j^{\prime} \mid j^{\prime} \in \bar{m}\right.$, and $\left.j^{\prime} \neq i\right\}$, and the choice of lth in case 3 can be designed as $l=\min \left\{l^{\prime} \mid l^{\prime} \in \bar{m}\right.$, and $\left.l^{\prime} \neq i, j\right\}$, but the choice is not the one and only.

Step 3. The procedure of the dynamic behaviors can be explained as:

Given the initial state $x(0)$, we can judge which region that $x(0)$ locates in. Without loss of generality, we can assume that $x(0)$ is contained in region $\hat{\Omega}_{i}$. Also the distance between the initial state and the equilibrium of the system $(0,0)$ can be defined as $g(x)=\sqrt{x^{\prime} P x}$, where $P$ is the positive definite matrix, which can be solved from LMI (3), and satisfied $g\left(x_{0}\right) \geq L(t)$. Based on the switching rules above, we can check the further dynamic behavior of the system by following two issues:

(1) If $g(x) \geq L(t)$ holds, the ith subsystem is activated. Due to the separation of $\hat{\Omega}_{i}$, we can see that $g(x)$ 
decreases in $\hat{\Omega}_{i}$ by using the analysis method as[6], so we have $g(x)_{\max }=\sqrt{x^{\prime}(0) P x(0)}$. If $g(x)$ decreases small enough and makes $g(x)<L(t)$ hold in the same region, then turn to (2). Else, if the condition $g(x) \geq L(t)$ is consistently fulfilled in $\hat{\Omega}_{i}$, when the orbit cross the boundary of $\hat{\Omega}_{i}$ and $\hat{\Omega}_{i j}$, the switching does not occur, and $g(x)$ decreases as mentioned above. While $g(x)<L(t)$ in a certain moment holds in the region $\hat{\Omega}_{i j}$, then turn to (2). Otherwise, the orbit goes through the boundary of $\hat{\Omega}_{i j}$ and $\hat{\Omega}_{i}$, another cycle begins.

(2) If $g(x)<L(t)$ holds and $x(t)$ locates in region $\hat{\Omega}_{i}$, then switching rule guides the system switching to $\mathrm{jth}$ subsystem, and $g(x)$ would increase within one or several regions until $g(x) \geq L(t)$ happens, and the procedure turns to $(1)$, here $g(x)<g(x)_{\max }$. If $x(t)$ locates in region $\hat{\Omega}_{i j}$, the system would switch from the ith subsystem to lth subsystem if there are more than two subsystems, then $g(x)$ would increase within one or several regions until $g(x) \geq L(t)$ happens, and the procedure turns to (1), we also have $g(x)<g(x)_{\max }$. But for those dual subsystems, the switching does not occur, and $g(x)$ decreases until the orbit goes into region $\hat{\Omega}_{j}$, still $g(x)<g(x)_{\max }$, another cycle begins.

Additional, if the initial state $x(0)$ satisfies $g\left(x_{0}\right)<L(t)$, it turn to (2), and similar course occurs. Only difference between them rests with $g(x)_{\max }=M$, which is predefined.

Remark 3. Both theoretical analysis and simulation show that $g(x)=\sqrt{x^{\prime} P x}$ is not the sole definition, others definition can also work, such as $g(x)=\|x\|_{2}$, it will be showed in the example 2 below.

Step 4. By the analysis above, we can conclude: For every linear subsystem mentioned in (2), the orbit of state is simple, it could be stable or unstable. When the designed rules are infused into the switching system, the diversity of the dynamic system specifically presents in another way. Based on the switching rules, the state orbits goes through different regions along with a fixed direction of rotation, and the distance from orbits to equilibrium iterates, the boundary of orbits is determined by the initial state and extreme value of $L(t)$. Hence, the state orbits are folded and stretched repeatedly in a given area,so it is able to generate chaos or chaos-like behaviors from non chaotic subsystems. $\square$.

\subsection{Chaotification Controller Design}

We will extend the Proposition 2.1 to the forced system by designing state feedback controllers in this section. The controllers can be:

$$
u(t)=K_{\sigma} x(t)
$$

We have the following result:

Proposition 2.2. If there exists a serial of matrices $Q_{i}$, and $\alpha_{1}, \cdots, \alpha_{m} \in(0,1), \sum_{i=1}^{m} \alpha_{i}=1$, a symmetric matrix $P>0$, such that the following LMI holds

$$
\bar{A}^{T} P+P \bar{A}+\bar{Q}^{T}+\bar{Q}<0
$$

where, $\bar{A}=\sum_{i=1}^{m} \alpha_{i} A_{i}, \bar{Q}=\sum_{i=1}^{m} \alpha_{i} Q_{i}$, and satisfying the structure requirement as mentioned in 2.1. Then, there exists switching rules and state feedback controllers

$$
K_{i}=P^{-1} Q_{i}
$$

making system(1) generate chaos or chaos-like behavior under the switching rules.

Remark 4. It's easy to see that the set of controllers is not unique. So it provides us with many choices. We can design suitable controllers according to concrete demands and applications. A reasonable design procedure could be concluded as follow.

Step 1. Choose working area based on the specific requirement;

Step 2. Figure out LMIs and solve them;

Step 3. Gain state feedback controllers;

Step 4. Separate switching regions;

Step 5. Design switching rules;

Step 6. Check and adjust.

\section{Numerical Simulation}

In this section, two examples are provided to illustrate the effective of the theoretical analysis and design. A class of 2-dimension switching control systems are considered. Example 3.1. Consider the autonomous switching systems(2) with

$$
\begin{gathered}
A_{1}=\left[\begin{array}{cc}
-1 & 10 \\
-1 & -0.8
\end{array}\right] \\
A_{2}=\left[\begin{array}{cc}
-0.4 & 1 \\
-6 & 1
\end{array}\right] \\
A_{3}=\left[\begin{array}{cc}
0.8 & 1 \\
-10 & -0.6
\end{array}\right]
\end{gathered}
$$

Given the initial state $x(0)=(0.01,0.02)$, the dynamic behaviors of subsystems show in Fig.1, Fig.2, Fig3.

When $\alpha_{1}=0.3, \alpha_{2}=0.3, \alpha_{3}=0.4$, we get

$$
\bar{A}=0.3 A_{1}+0.3 A_{2}+0.4 A_{3}=\left[\begin{array}{cc}
-0.10 & 3.70 \\
-6.10 & -0.18
\end{array}\right]
$$

It is a Hurwitz convex combination. By solving the LMI, we obtain

$$
P=\left[\begin{array}{ll}
1.9442 & 0.0251 \\
0.0251 & 1.1868
\end{array}\right]
$$




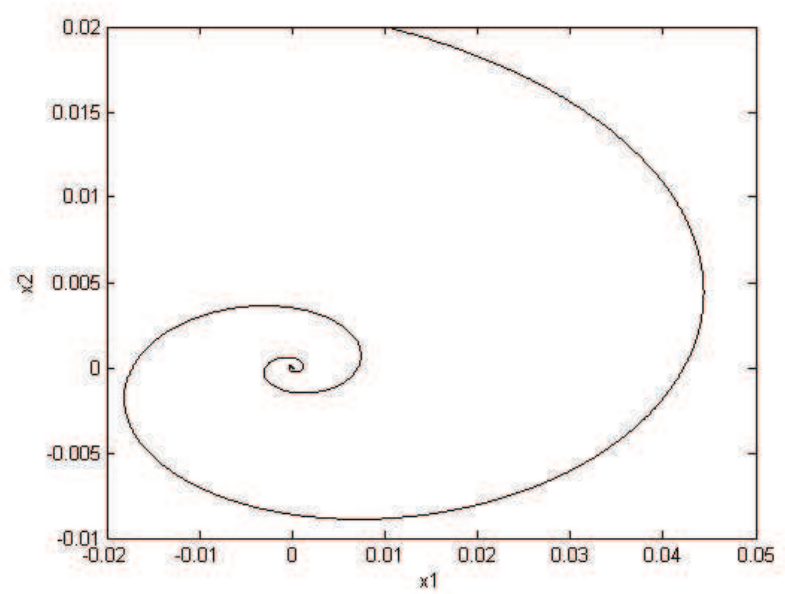

Fig. 1: Behavior of the subsystem 1 with initial point $(0.01,0.02)$.

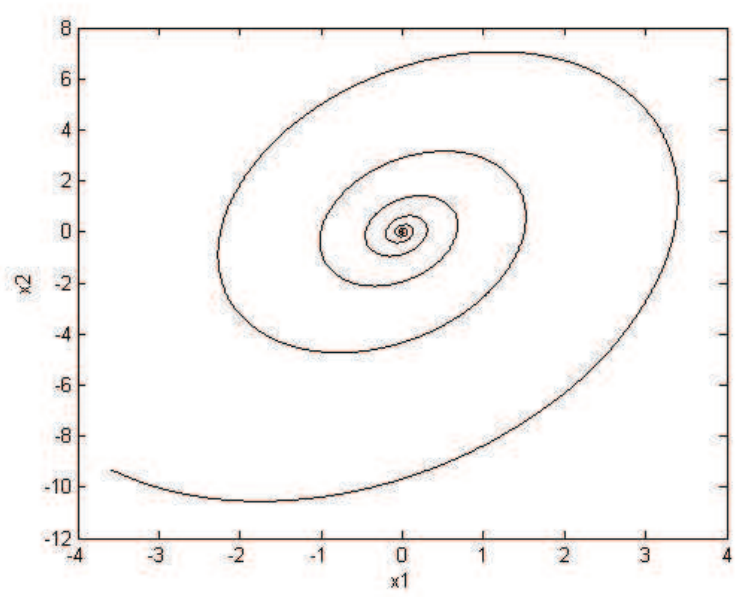

Fig. 2: Behavior of the subsystem 2 with initial point $(0.01,0.02)$.

According to Proposition 2.1., we can separate the plane as:

$$
\begin{array}{r}
\hat{\Omega}_{1}=\left\{\left(x_{1}, x_{2}\right) \mid\left(x_{1}+0.07 x_{2}\right)\left(x_{1}+5.79 x_{2}\right)<0\right\} ; \\
\hat{\Omega}_{2}=\left\{\left(x_{1}, x_{2}\right) \mid\left(x_{1}-9.21 x_{2}\right)\left(x_{1}-7.66 x_{2}\right)<0\right\} ; \\
\hat{\Omega}_{3}=\left\{\left(x_{1}, x_{2}\right) \mid\left(x_{1}-0.23 x_{2}\right)\left(x_{1}-0.04 x_{2}\right)<0\right\} ; \\
\hat{\Omega}_{12}=\left\{\left(x_{1}, x_{2}\right) \mid\left(x_{1}-9.21 x_{2}\right)\left(x_{1}+5.79 x_{2}\right) \geq 0\right\} ; \\
\hat{\Omega}_{23}=\left\{\left(x_{1}, x_{2}\right) \mid\left(x_{1}-0.23 x_{2}\right)\left(x_{1}-7.66 x_{2}\right) \leq 0\right\} ; \\
\hat{\Omega}_{31}=\left\{\left(x_{1}, x_{2}\right) \mid\left(x_{1}+0.07 x_{2}\right)\left(x_{1}-0.04 x_{2}\right) \leq 0\right\} . \\
\text { with } g(x)=\sqrt{x^{\prime} P x}, L(t)=5|\sin (w t)|, w=0.1 \pi .
\end{array}
$$

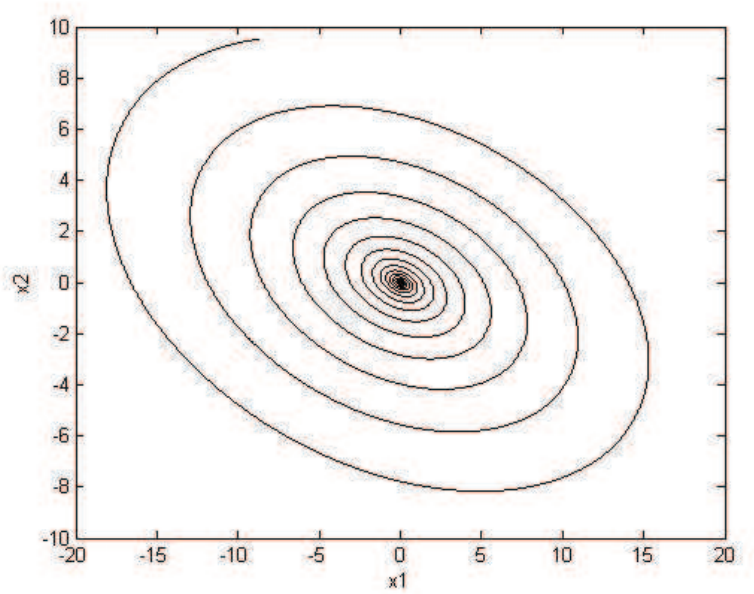

Fig. 3: Behavior of the subsystem 3 with initial point $(0.01,0.02)$.

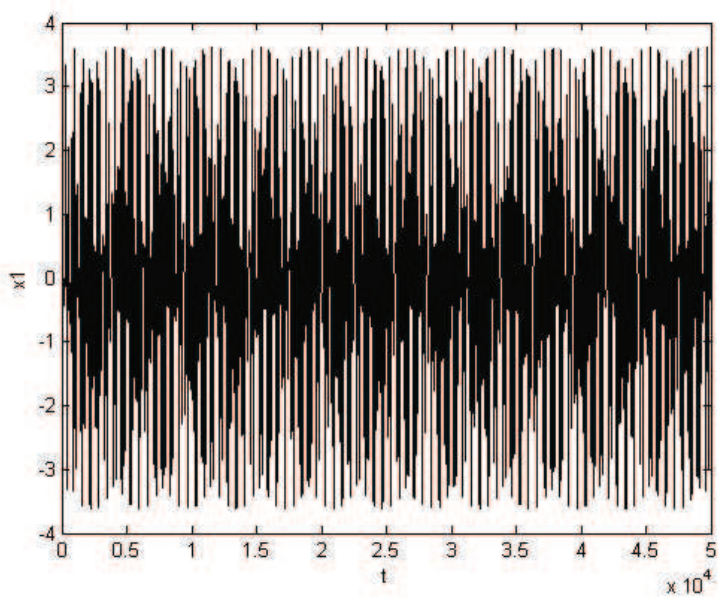

Fig. 4: Phase portrait $\mathrm{x} 1$ of switching system with initial point $(0.01,0.02)$.

The phase portraits and state orbit of the system(2) with parameters above are showed in Fig.4, Fig.5, Fig.6., respectively.

Example 3.2. Consider the switching systems(1) with

$$
\begin{gathered}
A_{1}=\left[\begin{array}{ll}
3 & 2 \\
6 & 4
\end{array}\right] \\
A_{2}=\left[\begin{array}{cc}
5 & 1 \\
-2 & 3
\end{array}\right]
\end{gathered}
$$

By solving LMI, we get a set of feasible solution:

$$
P=\left[\begin{array}{cc}
0.2258 & -0.0271 \\
-0.0271 & 1.2684
\end{array}\right]
$$




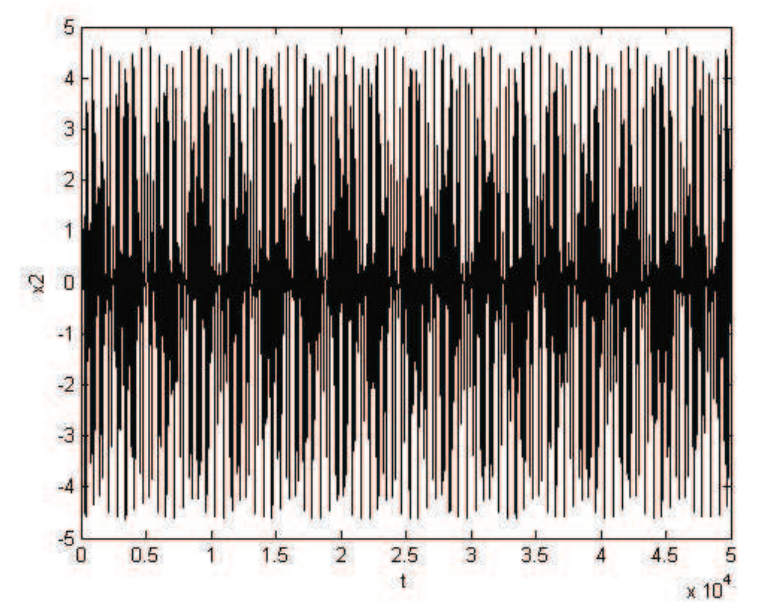

Fig. 5: Phase portrait $\mathrm{x} 2$ of switching system with initial point $(0.01,0.02)$.

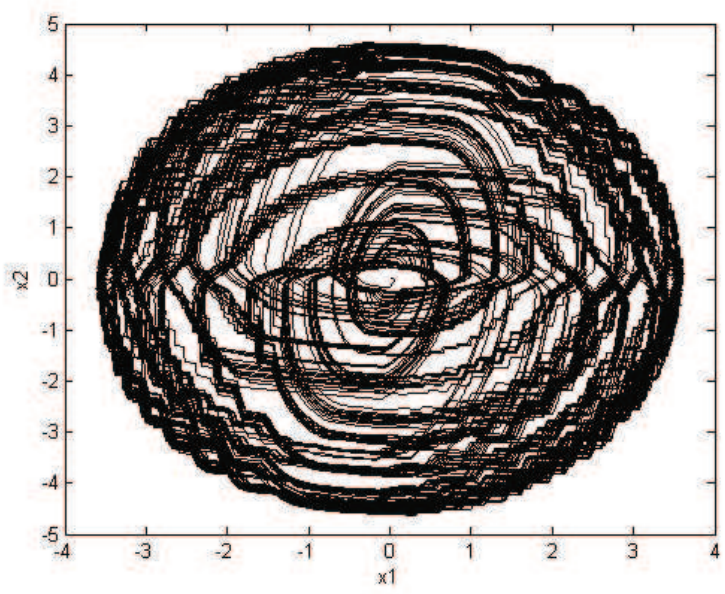

Fig. 6: State orbit of switching system with initial point $(0.01,0.02)$.

$$
\begin{aligned}
& Q_{1}=\left[\begin{array}{cc}
-0.7135 & 1.9365 \\
0.1058 & -0.2186
\end{array}\right] \\
& Q_{2}=\left[\begin{array}{cc}
-1.0206 & 0.2963 \\
0.1196 & -0.0281
\end{array}\right]
\end{aligned}
$$

Then, we obtain

$$
\begin{aligned}
& K_{1}=\left[\begin{array}{cc}
-4.0 & 8.0 \\
-7.0 & -4.8
\end{array}\right] \\
& K_{2}=\left[\begin{array}{cc}
-4.4 & 1.0 \\
1.0 & -2.6
\end{array}\right]
\end{aligned}
$$

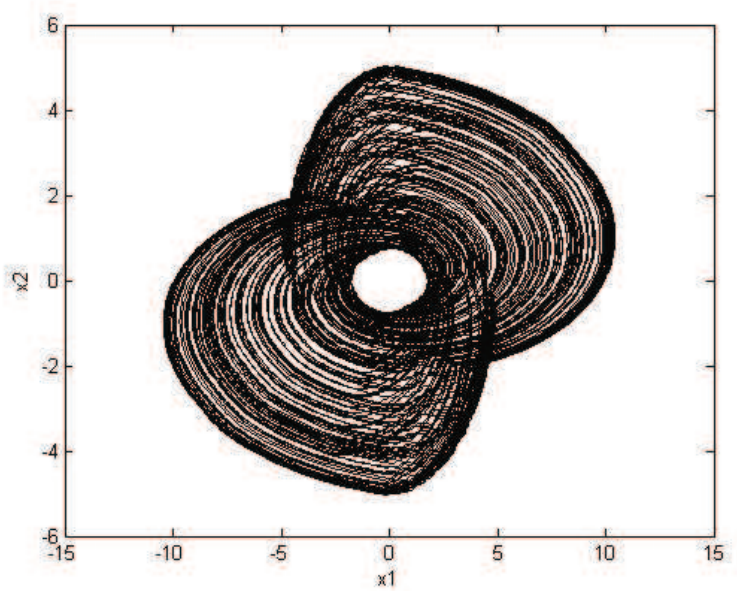

Fig. 7: State orbit of switching system with initial point(1,1).

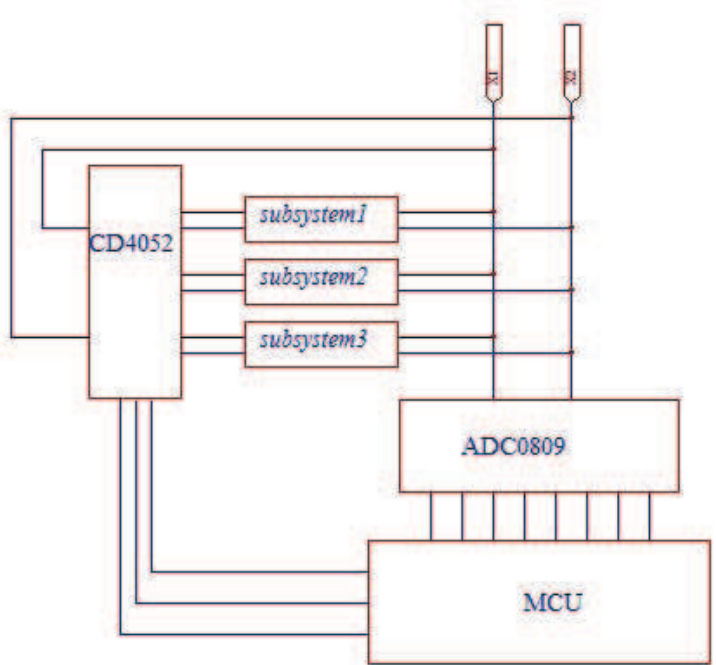

Fig. 8: Circuit realization of switching system.

And switching regions can be divided as

$$
\begin{aligned}
\hat{\Omega}_{1}= & \left\{\left(x_{1}, x_{2}\right) \mid\left(x_{1}-4.58 x_{2}\right)\left(x_{1}-0.61 x_{2}\right)>0\right\} ; \\
\hat{\Omega}_{2}= & \left\{\left(x_{1}, x_{2}\right) \mid\left(x_{1}-2.02 x_{2}\right)\left(x_{1}-3.21 x_{2}\right)<0\right\} ; \\
\hat{\Omega}_{12}= & \left\{\left(x_{1}, x_{2}\right) \mid\left(x_{1}-4.58 x_{2}\right)\left(x_{1}-3.21 x_{2}\right) \leq 0\right. \\
& \left.\bigcup\left(x_{1}-0.61 x_{2}\right)\left(x_{1}-2.02 x_{2}\right) \leq 0\right\} .
\end{aligned}
$$

with $g(x)=\|x\|_{2}, L(t)=5|\sin (w t)|, w=0.1 \pi$.

Then the state orbit of switching systems with feedback controllers is showed in Fig.7. 


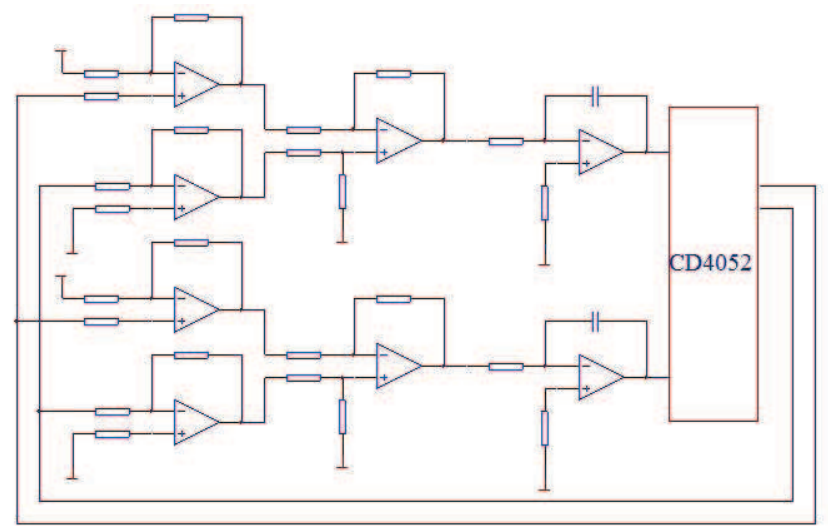

Fig. 9: Circuit realization of a subsystem.

\section{Circuitry Realization}

In this section, we contrive a circuit to illustrate the chaotification of switching system for Example 1 to further confirm the reality and validity of designed method. The circuit of the system is partly showed in Fig.8 and Fig.9. It demonstrates that generation of chaos or chaos-like behavior by switching method is reasonable to be realized especially in electrical circuit.

\section{Conclusions}

A novel approach to generate chaos by designing switching rules in 2-dimension switching systems has been proposed in this paper, and corresponding state feedback controllers also have been obtained. Two examples were provided to illustrate the effectivity of the method. And a possible circuit was also provided, and the potential application of the method could be realized easily by this means. Additionally, further work is how to extend the method to higher dimension and how to deal with subsystems with delays.

\section{Acknowledgement}

The authors would like to thank the anonymous referee and editors of the details and for helpful comments that improve this paper. This research is funded by China Scholarship Council, the Fundamental Research Funds for the Central Universities (103.1.2 E022050205).

\section{References}

[1] S. Sahin , Cüneyt Güzelis, IEEE Antennas and Propagation Magazine, 52, 222-233 (2010).
[2] A. Ikhlef and N. Mansouri, 2008 5th International multiconference on system, signals and devices, 1-5 (2008).

[3] D. Q. Wang, D. C. Yu, and W. G. Bi, Proceedings of the 6th world conference on intelligent control and automation, Dalian, China, 914-919 (2006).

[4] X. Z. Liu, K. L. Teo, H. T. Zhang, G. R. Chen, Chaos solitons \& fractals, 30, 725-733 (2006).

[5] M. S. Branicky, IEEE Trans. On Automatic Control, 43, 475482 (1998).

[6] Y. P. Zhang, X. Z. Liu, H. Zhu, S. M. Zhong, Applied mathematics and computation, 190, 1258-1266 (2007) .

[7] L. L. Xiong, S. M. Zhong, M. Ye, Chaos solitons \& fractals, 42, 1800-1811 (2009).

[8] D. Y. Liu, S. M. Zhong, X. Z. Liu, Y. Q. Huang, Mathematics and Computers in Simulation, 80, 436-448 (2009). 


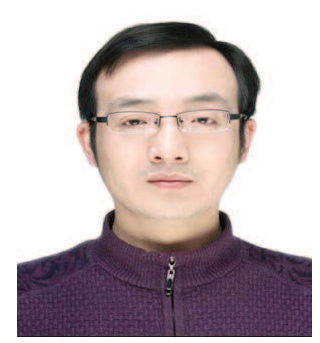

Zhang Yuping received the $\mathrm{PhD}$ degree in School of Automation Engineering, University of Electronic Science and Technology of China. $\mathrm{He}$ is currently an Associate Professor of the School of Automation Engineering at University of Electronic Science and

Technology of China. His research interests are in the areas of dynamic behaviors of complex systems.

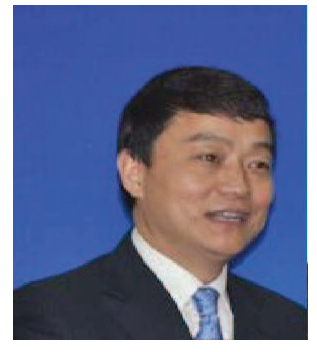

Zhu Hong received the $\mathrm{PhD}$ degree from Operational Research and Cybernetics, Sichuan University. He is currently a Professor of the School of Automation Engineering at University of Electronic Science and Technology of China. His research works focus on applied mathematics and information science, and more than 50 scholar papers were published in these years.

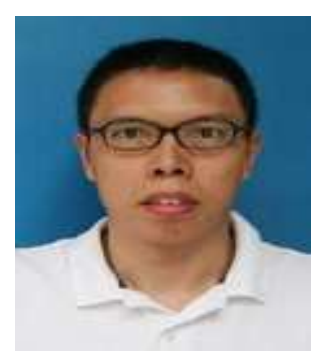

Zeng Yong received his B.E. degree in Precision Instrument from Harbin University of Science and Technology, Harbin, China, and M.E. degree in Pattern Recognition and Intelligent System from University of Electronic Science and Technology of China, Chengdu, China, and the Ph.D. degree in control theory and control engineering from Shanghai Jiaotong University, Shanghai, China. He is currently a associate professor at the School of Automation Engineering, University of Electronic Science and Technology of China, Chengdu, China. His research interests include intelligent information processing, intelligent control theory and applications, computer vision and pattern recognition. 